\title{
Convection - Diffusion - Radiation Heat and Mass Transfer to a Sphere Accompanied by a Surface Exothermal Chemical Reaction
}

\author{
Gheorghe Juncu \\ POLITEHNICA University Bucharest \\ Department of Chemical and Biochemical Engineering \\ Polizu 1, 011061 Bucharest \\ Romania \\ E-mail: juncu.gheorghe@yahoo.com \\ juncu@cael.pub.ro
}

\begin{abstract}
The steady-state, coupled heat and mass transfer from a fluid flow to a sphere accompanied by an exothermal catalytic chemical reaction on the surface of the sphere is analysed taking into consideration the effect of thermal radiation. The flow past the sphere is considered steady, laminar and incompressible. The radiative transfer is modeled by $P_{0}$ and $P_{1}$ approximations. The mathematical model equations were discretized by the finite difference method. The discrete equations were solved by the defect correction - multigrid method. The influence of thermal radiation on the sphere surface temperature, concentration and reaction rate was analysed for three parameter sets of the dimensionless reaction parameters. The numerical results show that only for very small values of the Prater number the effect of thermal radiation on the surface reaction is not significant.
\end{abstract}

Keywords: heat transfer; mass transfer; convection-radiation; surface reaction; diffusion approximation; finite difference. 


\section{List of symbols}

$a$ - radius of the sphere, $\mathrm{m}$

$B$ - dimensionless adiabatic heat rise (Prater number), ( - $\left.\Delta H_{R} D C_{\infty}\right) /\left(\lambda T_{\infty}\right)$

$\mathfrak{B}$ - dimensionless group, $\mathfrak{B}=\beta a$

$c_{P}$ - heat capacity, $\mathrm{J} /(\mathrm{kg} \mathrm{K})$

$C$ - concentration of reactant, $\mathrm{kg} / \mathrm{m}^{3}$

$d$ - diameter of the sphere, $d=2 a, \mathrm{~m}$

$D$ - diffusion coefficient, $\mathrm{m}^{2} / \mathrm{s}$

$E$ - activation energy of the chemical reaction, $\mathrm{J} / \mathrm{mol}$

$\mathcal{E}$ - dimensionless group, $\mathcal{E}=\frac{\varepsilon}{(2-\varepsilon)}$

$G$ - dimensionless directed - integrated intensity of the radiation, $G=\frac{G^{*}}{4 n^{2} \sigma T_{\infty}^{4}}$

$G^{*}$ - dimensional directed - integrated intensity of the radiation, $\mathrm{W} / \mathrm{m}^{2}$

$k_{a}$ - absorption coefficient, $\mathrm{m}^{-1}$

$k_{0}$ - pre-exponential factor, $\mathrm{m} / \mathrm{s}$

$K$ - dimensionless group, $K=k_{a} a$

$n$-index of refraction, dimensionless

$\mathrm{Nu}$ - average Nusselt number defined by relation (13), dimensionless

$N u_{\theta}-$ local Nusselt number defined by relations (14), dimensionless

$\operatorname{Pr}-$ Prandtl number, $\mathrm{Pe}=\mu / \rho \alpha$, dimensionless

$q_{r}$ - dimensionless radiative heat flux vector

$R e-$ sphere Reynolds number, $R e=U_{\infty} d \rho / \mu$, dimensionless

$R d_{0}$ - Rosseland radiation - conduction parameter, dimensionless

$R d_{1}$ - radiation - conduction parameter for $P_{1}$ approximation, dimensionless

$R_{G}-$ gas constant, J / (mol K)

$r$-dimensionless radial coordinate, $r^{*} / a$, in spherical coordinate system

$r^{*}$ - radial coordinate in spherical coordinate system, $\mathrm{m}$

Sc - Schimdt number, $S c=\mu / \rho D$, dimensionless

$S h$ - average Sherwood number defined by relation (13), dimensionless

$S h_{\theta}-$ local Sherwood number defined by relation (14), dimensionless 
$T$ - temperature, $\mathrm{K}$

$U_{\infty}$ - velocity far away from the sphere, $\mathrm{m} / \mathrm{s}$

$V_{R}$ - dimensionless radial velocity component

$V_{\theta}$ - dimensionless tangential velocity component

$X-$ conversion of reactant, dimensionless

$Y$ - dimensionless concentration of reactant, $C / C_{\infty}$

$Z$ - dimensionless temperature, $T / T_{\infty}$

\section{Greek symbols}

$\alpha$ - thermal diffusivity, $\mathrm{m}^{2} / \mathrm{s}$

$\beta$ - extinction coefficient (total attenuation factor), $\mathrm{m}^{-1}$

$\beta_{R}$ - Rosseland mean extinction coefficient, $\mathrm{m}^{-1}$

$\gamma$-dimensionless activation energy (Arrhenius number), $E / R_{G} T_{\infty}$

$\Delta H_{R}$ - heat of reaction, $\mathrm{J} / \mathrm{kg}$

$\varepsilon$ - emissivity coefficient

$\eta_{e}-$ external effectiveness factor defined by relation (12), dimensionless

$\lambda$ - thermal conductivity, $\mathrm{W} /(\mathrm{m} \mathrm{K})$

$\mu$ - dynamic viscosity, $\mathrm{kg} /$ (m s)

$\theta$ - polar angle in spherical coordinate system, rad

$\rho$-density, $\mathrm{kg} / \mathrm{m}^{3}$

$\sigma$ - Stefan - Boltzman constant, W / $\left(\mathrm{m}^{2} \mathrm{~K}^{4}\right)$

$\varphi-$ reaction parameter, $\varphi=\frac{a k_{0} \exp (-\gamma)}{D}$, dimensionless

\section{Subscripts}

$\infty$ - refers to a large finite distance from the center of the sphere

$s$ - refers to the surface of the sphere 


\section{Introduction}

Mass and heat transfer with surface chemical reactions have many applications in aerothermochemistry, environmental, industrial and life science processes. Examples of these applications are the catalytic combustion of hydrogen, methane, natural gas and volatile organic compounds (VOCs) and entries in Earth's atmosphere. The catalytic combustion of methane or natural gas is one of the most promising solutions for rational and clean use of fossil energies. The VOCs emission from industrial processes and vehicle exhausts is considered a severe air pollutant. Catalytic oxidation is an effective way for reducing the emissions of VOCs.

The articles published until 1965 about the convection - diffusion mass / heat transfer accompanied by a surface chemical reaction were reviewed by Chung [1]. Due to the topic of this work, the citation from this section is restricted to external flow problems. Fox and Libby [2] present a systematically analysis for different laminar boundary layers with surface reaction. For chemical reactions of any order, the solution for the surface concentration is given by an integral equation which can be solved numerically. Freeman and Simpkins [3] applied Mellin transform technique for the diffusion of species in a boundary layer of Falkner - Skan type with surface chemical reaction. Mihail [4] derived a general superposition integral equation for calculating the local concentration of a catalytic surface for either laminar or turbulent boundary layer flows with and without pressure gradients over the surface. This technique was extended to non - Newtonian fluids in [5] and to bluff bodies in [6]. Gupalo and Ryazantsev [7] derived an approximate solution for the steady-state mass and heat transfer with first - order surface chemical reaction from a moving solid sphere at small finite Péclet and Reynolds numbers. The problem is solved by means of matching inner and outer asymptotic expansions in terms of the Péclet number. The isothermal convection - diffusion mass transfer to a sphere with a non-linear chemical reaction rate on the surface of the sphere was analysed in [8] and [9] using the boundary layer formalism.

Schefer [10] analysed the combustion of $\mathrm{H}_{2}$ on catalytic surfaces. The surface catalytic combustion of methane was investigated by Markatou et al. [11]. Elperin and Fominykh [12, 13] and Elperin et al. [14] solved the case of mass transfer with a first-order chemical reaction on the wall. Merkin [15] studied the case of homogeneous-heterogeneous isothermal chemical reaction. In $[10-15]$ the boundary layer approximation was used to model the process. 
The coupled heat and mass transfer to a sphere with a first-order, non-isothermal chemical reaction on the surface of the sphere was analysed numerically by Juncu and Mihail [16] without the boundary layer assumptions. Paterson [17] investigated the case of isothermal surface reaction in spherical geometry using the film model for mass transfer.

Lindberg and Schmitz [18] analysed the multiplicity of the steady state solutions for laminar boundary layer flow with chemical reaction on surfaces of the wedge type. It is shown that the usual boundary layer equations have a unique solution while the mathematical model that takes into account the longitudinal conduction of heat in the solid plate admits multiple solutions. The results obtained in [18] were extended to a parabolic cylinder surface in [19]. The multiplicity of the steady states for boundary layer flow with surface chemical reaction was also investigated by Mihail and Teodorescu [20] and Linan and Trevino [21].

In the articles mentioned previously the effect of thermal radiation on the surface chemical reaction was not analysed. A first step in this direction can be viewed in [22]. The aim of the present work is to complete the investigation from [22] concerning the effect of thermal radiation on forced convection heat and mass transfer to a sphere in the presence of an exothermic, catalytic, surface chemical reaction. The flow past the sphere is considered steady, laminar and incompressible. The diffusion approximations of radiative transfer equation (RTE) $\left(P_{0}\right.$ and $\left.P_{1}\right)$ $[23,24,25]$ were used to model the radiative transfer.

This paper is organized as follows. The next section describes the mathematical model of the problem. Section Method of solution presents the numerical algorithm. The numerical simulations and the results obtained are presented in section Results. The concluding remarks of this work are briefly mentioned in the final section.

\section{Mathematical model}

Let us consider a sphere placed in a laminar, incompressible, steady flow of a Newtonian fluid. The flow configuration and coordinate system are shown in figure 1 . The diameter of the sphere $d$ is assumed considerably larger than the mean free path of the surrounding fluid. The free stream velocity, temperature and concentration are $U_{\infty}, T_{\infty}$ and $C_{\infty}$. On the surface of the sphere an exothermic, first-order catalytic chemical reaction occurs. The physical properties are constant and 
isotropic. The effects of buoyancy, viscous dissipation and work done by pressure forces are considered negligible.

For the assumptions discussed previously, the dimensionless energy and chemical species balance equations for a participating medium (the radius of the sphere $a$ is considered the length scale and the free stream velocity $U_{\infty}$ the velocity scale), expressed in dimensionless spherical coordinate system $(r, \theta)$, are:

- Chemical species balance equation

$$
\frac{\operatorname{ReS} C}{2}\left(V_{R} \frac{\partial Y}{\partial r}+\frac{V_{\theta}}{r} \frac{\partial Y}{\partial \theta}\right)=\frac{1}{r^{2}} \frac{\partial}{\partial r}\left(r^{2} \frac{\partial Y}{\partial r}\right)+\frac{1}{r^{2} \sin \theta} \frac{\partial}{\partial \theta}\left(\sin \theta \frac{\partial Y}{\partial \theta}\right)
$$

- Energy balance equation

$$
\frac{R e P r}{2}\left(V_{R} \frac{\partial Z}{\partial r}+\frac{V_{\theta}}{r} \frac{\partial Z}{\partial \theta}\right)=\frac{1}{r^{2}} \frac{\partial}{\partial r}\left(r^{2} \frac{\partial Z}{\partial r}-r^{2} q_{r, r}\right)+\frac{1}{r^{2} \sin \theta} \frac{\partial}{\partial \theta}\left(\sin \theta \frac{\partial Z}{\partial \theta}-\sin \theta q_{r, \theta}\right)
$$

where $q_{r, r}$ and $q_{r, \theta}$ are the dimensionless normal and tangential components of the radiative heat flux vector $q_{r}, q_{r}=q_{r}\left(q_{r, r}, q_{r, \theta}\right)$.

The boundary conditions to be satisfied by the dimensionless concentration and temperature are:

- $\quad$ Symmetry axis $(\theta=0, \pi)$;

$$
\frac{\partial Z}{\partial \theta}=0, \frac{\partial Y}{\partial \theta}=0
$$

- $\quad$ Surface of the sphere $(r=1)$;

$$
\begin{gathered}
\frac{\partial Z}{\partial r}-q_{r, r}=-B \varphi Y \exp \left[\gamma\left(1-\frac{1}{Z}\right)\right] \\
\frac{\partial Y}{\partial r}=\varphi Y \exp \left[\gamma\left(1-\frac{1}{Z}\right)\right]
\end{gathered}
$$

- Free stream $(r \rightarrow \infty)$;

$$
Z=1.0, Y=1.0
$$

The computation of the radiative heat flux implies the solving of RTE. RTE is an integrodifferential equation very difficult to solve. For this reason, approximate mathematically less complicated but accurate models have been developed. The key quantity used to select the approximate mathematical model for RTE is the optical thickness. For the present problem, the optical thickness of the medium is, theoretically, infinite. In this case, the diffusion models approximate accurately RTE. The diffusion models used in this work are: $P_{0}$ (Rosseland) and $P_{1}$. The fluid is assumed to be a gray, emitting, absorbing and isotropic scattering medium. 


\section{$P_{0}($ Rosseland $)$ approximation}

The radial and tangential components of the dimensionless radiative heat flux vector given by Rosseland approximation [24] read as:

$$
q_{r, r}=-R d_{0} Z^{3} \frac{\partial Z}{\partial r}, q_{r, \theta}=-R d_{0} Z^{3} \frac{\partial Z}{\partial \theta} .
$$

where $R d_{0}$ is the Rosseland radiation - conduction parameter, $R d_{0}=\frac{16}{3} \sigma T_{\infty}^{3} /\left(\lambda_{f} \beta_{R}\right)$. If we denote, for the simplicity of writing, $f=R d_{0} Z^{3}$, equation (2) can be rewritten as

$$
\frac{R e P r}{2}\left(V_{R} \frac{\partial Z}{\partial r}+\frac{V_{\theta}}{r} \frac{\partial Z}{\partial \theta}\right)=\frac{1}{r^{2}} \frac{\partial}{\partial r}\left[r^{2}(1+f) \frac{\partial Z}{\partial r}\right]+\frac{1}{r^{2} \sin \theta} \frac{\partial}{\partial \theta}\left[\sin \theta(1+f) \frac{\partial Z}{\partial \theta}\right]
$$

\section{$P_{1}$ approximation}

For $P_{1}$ approximation [24], the dimensionless radiative heat flux vector $q_{r}\left(q_{r, r}, q_{r, \theta}\right)$ satisfies the equation

$$
\frac{1}{r^{2}} \frac{\partial}{\partial r}\left(r^{2} q_{r, r}\right)+\frac{1}{r^{2} \sin \theta} \frac{\partial}{\partial \theta}\left(\sin \theta \frac{\partial q_{r, \theta}}{\partial \theta}\right)=-K^{2} R d\left(Z^{4}-G\right)
$$

where $G$ is the dimensionless directed - integrated intensity of the radiation, $K=k_{a} a, R d=$ $4 \sigma n^{2} T_{\infty}^{3} /\left(k_{a} \lambda\right)$. Substituting equation (6) into equation (2), it results:

$$
\frac{R e P r}{2}\left(V_{R} \frac{\partial Z}{\partial r}+\frac{V_{\theta}}{r} \frac{\partial Z}{\partial \theta}\right)=\frac{1}{r^{2}} \frac{\partial}{\partial r}\left(r^{2} \frac{\partial Z}{\partial r}\right)+\frac{1}{r^{2} \sin \theta} \frac{\partial}{\partial \theta}\left(\sin \theta \frac{\partial Z}{\partial \theta}\right)-K^{2} R d\left(Z^{4}-G\right)
$$

The dimensionless directed - integrated intensity of radiation $G$ verifies the equation [24]:

$$
\frac{1}{r^{2}} \frac{\partial}{\partial r}\left(r^{2} \frac{\partial G}{\partial r}\right)+\frac{1}{r^{2} \sin \theta} \frac{\partial}{\partial \theta}\left(\sin \theta \frac{\partial G}{\partial \theta}\right)-3 \mathfrak{B} K\left(G-Z^{4}\right)=0
$$

where $\mathfrak{B}=\beta$ a.

The boundary conditions to be satisfied by $G$ are [24]:

- $\quad$ Symmetry axis $(\theta=0, \pi)$;

$$
\frac{\partial G}{\partial \theta}=0
$$

- $\quad$ Surface of the sphere $(r=1)$;

$$
-\frac{\partial G}{\partial r}+\frac{3}{2} \mathfrak{B} \mathcal{E}\left(G-Z^{4}\right)=0
$$

- Free stream $(r \rightarrow \infty)$; 


$$
G=Z^{4}=1.0
$$

where $\mathcal{E}=\frac{\varepsilon}{(2-\varepsilon)}$. The boundary condition (9c) considers radiative equilibrium at free stream. For $P_{1}$ approximation, the dimensionless radiative heat flux is given by [24],

$$
q_{r}=-1 / \mathfrak{B} \operatorname{grad} G .
$$

The physical quantities of interest are the sphere surface dimensionless average temperature, concentration or conversion, $\bar{Z}_{s}, \bar{Y}_{s}, \bar{X}_{s}$, the external effectiveness factor $\eta_{e}$, and the average Nusselt / Sherwood number, Nu/Sh. The sphere surface dimensionless average temperature, concentration and conversion $\bar{Z}_{s}, \bar{Y}_{s}, \bar{X}_{s}$, were computed with the relations:

$$
\begin{gathered}
\bar{Z}_{s}=\left.\frac{1}{2} \int_{0}^{\pi} Z\right|_{r=1} \sin \theta d \theta \\
\bar{Y}_{s}=\left.\frac{1}{2} \int_{0}^{\pi} Y\right|_{r=1} \sin \theta d \theta \\
\bar{X}_{s}=1-\bar{Y}_{s} .
\end{gathered}
$$

The external effectiveness factor was calculated with the relation:

$$
\eta_{e}=\left.\frac{1}{2} \int_{0}^{\pi} Y\right|_{r=1} \exp \left[\gamma\left(1-\frac{1}{Z}\right)\right] \sin \theta d \theta
$$

Considering as driving force the difference $\left(\bar{Z}_{S}-1\right) /\left(1-\bar{Y}_{S}\right)$, the average Nusselt / Sherwood number based on the diameter of the sphere is given by:

$$
N u=\frac{1}{2} \int_{0}^{\pi} N u_{\theta} \sin \theta d \theta, S h=\frac{1}{2} \int_{0}^{\pi} S h_{\theta} \sin \theta d \theta
$$

where $N u_{\theta} / S h_{\theta}$ is the local Nusselt / Sherwood number. The local $N u$ / $S h$ number was computed with the relation:

- local Sherwood number

$$
S h_{\theta}=\left.\frac{2}{1-\bar{Y}_{S}} \frac{\partial Y}{\partial r}\right|_{r=1}
$$

- local Nusselt number

for $P_{0}$ approximation

$$
N u_{\theta}=-\left.\frac{2}{\overline{Z_{s}}-1}\left(1+R d_{0} Z_{s}^{3}\right) \frac{\partial Z}{\partial r}\right|_{r=1}
$$

for $P_{1}$ approximation

$$
N u_{\theta}=-\left.\frac{2}{\overline{Z_{s}}-1} \frac{\partial Z}{\partial r}\right|_{r=1}+\frac{R d_{1} K \varepsilon}{\overline{Z_{S}}-1}\left(Z^{4}-G\right)
$$




\section{Method of solution}

The mathematical model equations were solved numerically with the nested multigrid defect-correction method [26]. The mass and energy balance equations $(1,5,7)$ were discretized with the upwind and centered finite difference schemes (a double discretization required by the defect - correction iteration) on a vertex-centered grid, [27]. The spatial derivatives of the radiative transfer equation (6) were approximated by the centered finite difference scheme. Numerical experiments were made on meshes with the discretization steps, $\Delta \theta=\pi / 64, \Delta r=1 / 64, \Delta \theta=\pi$ / 128, $\Delta r=1 / 128$ and $\Delta \theta=\pi / 256, \Delta r=1 / 256$. The external boundary conditions (3d), (9c) and (14d) are assumed to be valid at a large but finite distance from the center of the sphere.

The defect - correction iteration was applied only to the discrete approximation of the mass and heat balance equations. Two multigrid cycles were used inside the defect - correction iteration step. The structure of the MG cycle is: 1) cycle of type V; 2) smoothing by alternating line Gauss Seidel method; 3) two smoothing steps are performed before the coarse grid correction and one after; 4) prolongation by bilinear interpolation for corrections; 5) restriction of residuals by full weighting. For $P_{1}$ approximation, the radiative transfer equations were solved by the multigrid method previously presented. Excepting the case $R e=0.1$, where the creeping flow solutions were used, the velocity field $\left(V_{R}, V_{\theta}\right)$ was calculated solving numerically the Navier-Stokes equations, $[26]$.

The error criteria employed are: the discrete $L_{2}$ norm of the residuals and the discrete $L_{\infty}$ norm of the difference between the numerical solutions of two consecutive defect - correction iterations are smaller than $10^{-8}$. The mesh independence of the $\mathrm{Nu} / \mathrm{Sh}$ number and dimensionless sphere average surface temperature and concentration is another accuracy test used in the present work.

Results that can be used to validate the accuracy of the present computations are not available in literature. A partial validation of the present accuracy computations consists of the numerical simulation of the physical mass and heat transfer (the boundary conditions on the surface of the sphere are $Z=Y=0$ ). The values obtained for the $N u$ and $S h$ numbers in this case agree better than $2 \%$ to the results calculated with the relations proposed by Clift et al. [28] (relation 3-49 for $R e=0.1$ and relation 5-25 for $R e>0.1$ ). 


\section{Results}

The present mathematical model takes into consideration three phenomena; convection diffusion, thermal radiation and surface chemical reaction. The influence of each phenomenon on the solution of the mathematical model is quantified by its dimensionless groups, i.e.: (1) convection - diffusion, $\operatorname{Pr}, \operatorname{Re}$ and $S c$; (2) radiative heat transfer, $\mathfrak{B}, \mathcal{E}, K$ and $R d$; (3) surface chemical reaction $B, \varphi$ and $\gamma$.

For steady, laminar, axisymmetric flow, $R e$ number takes values in the range $0.1 \div 200$, [29]. The numerical values considered for $\operatorname{Pr}$ and Sc numbers are, $\operatorname{Pr}=1.0$ and $S c=2.0$. The values of the radiative dimensionless groups $\mathfrak{B}, \mathcal{E}$ and $K$ are given by the values of $a, k_{a}, \beta$ and $\varepsilon$. The numerical results presented in this section were computed for $k_{a}=1 \mathrm{~m}^{-1}$ and $\beta=3 \mathrm{~m}^{-1}$, [24]. The numerical value assumed for the radius of the sphere and the emissivity are: $a=0.01 \mathrm{~m}$, and $\varepsilon=0.7$. The values for the Prater number, $B$, the dimensionless activation energy, $\gamma$ and the dimensionless reaction parameter, $\varphi$, were calculated starting from the information available in [10], [11], [30-35].

From the numerical experiments made, the results provided by three parameter sets of reaction dimensionless groups were selected to be presented in this work. This selection captures the salient features of the process.

The first set of dimensionless reaction parameters is: $B=0.85, \gamma=18.0, \varphi=0.06$. These values were computed for $T_{\infty}=298 \mathrm{~K}$ and large values of $C_{\infty}$. Considering $\lambda=0.03 \mathrm{~W} \mathrm{~m}^{-1} \mathrm{~K}^{-1}$, the value computed for $R d_{1}$ is $R d_{1}=200$. Based on the information presented in [24] $\left(\beta_{R}\right.$ is approximately ten times greater than $k_{a}$ ), the value considered for $R d_{0}$ is $R d_{0}=20$.

For this set of parameters, the influence of the $R e$ number on the sphere surface average dimensionless concentration, $\bar{Y}_{s}$, and temperature, $\bar{Z}_{s}$, is presented in figures 2 and 3, respectively. Figures 2 and 3 show that thermal radiation influences significantly the numerical solutions. When thermal radiation is neglected $(R d=0)$, chemical transformation is practically complete and the surface temperature increases significantly. In addition, for $R e>60$, the mathematical model exhibits multiple steady states. When thermal radiation is taken into consideration, the chemical transformation is negligible and the temperature variation is very small. All the radiation models employed in this work predict the same behaviour. The differences between the numerical results provided by these models are negligible. The effect of the thermal radiation is not influenced by 
the increase in $R e$. It must be also mentioned that for the no-radiation model, results similar to those computed for $R d_{1}=200 / R d_{0}=20$ were obtained for $\varphi=0.002$ (obviously, the other parameters have the same values).

For the second set of reaction dimensionless parameters the values of $B, \gamma$ and $\varphi$ are: $B=$ $0.05, \gamma=12.0$ and $\varphi=6.4$. These values were computed for $T_{\infty} \cong 400 \mathrm{~K}$ and small values of $C_{\infty}$. For $T_{\infty} \cong 400 \mathrm{~K}$ and $\lambda=0.048 \mathrm{~W} \mathrm{~m}^{-1} \mathrm{~K}^{-1}$, the value calculated for $R d_{1}$ is $R d_{1}=300$. As in the previous case, the value considered for $R d_{0}$ is ten times smaller than the value of $R d_{1}$, i.e. $R d_{0}=$ 30.

Figures 4 and 5 show the values of the sphere surface average dimensionless temperature, $\bar{Z}_{s}$, and concentration, $\bar{Y}_{S}$, for different radiation models and $R e$ in the range $0.1 \div 200$. For the second set of dimensionless parameters, the effect of thermal radiation on the numerical solutions is different compared to the previous case. As expected, for very small values of the Prater number, the effect of thermal radiation on the sphere dimensionless temperature $\bar{Z}_{s}$ is not significant (figure 4). The differences between the solutions of the different mathematical models are smaller than $5 \%$. The system can be considered practically isotherm for $P_{0}$ approximation.

The temperature profiles are different, depending on the radiation model. The temperature profile is the result of the competition between surface reaction, heat transfer and thermal radiation. Thermal radiation decreases the temperature on the surface of the sphere $\left(P_{1}<P_{0}\right)$ and implicitly the heat transfer rate and the surface reaction rate. The surface temperature increases with the increase in $R e$ if the surface reaction is the dominant phenomenon. If the heat transfer is the dominant phenomenon, the surface temperature decreases with the increase in $R e$. Note that the surface reaction rate also depends on the mass transfer rate.

Figure 5 shows that the increase in $R e$ increases the values of the sphere surface average dimensionless concentration regardless the radiation model. The increase in $\bar{Y}_{S}$ with the increase in $R e$ is due to the fact that the increase in the mass transfer rate is greater than the increase in the surface reaction rate. As mentioned previously, thermal radiation decreases the reaction rate. For this reason, for a given $R e$ value, the concentration on the surface of the sphere verifies the relation,

$$
\bar{Y}_{S}(R d=0)<\bar{Y}_{S}\left(R d_{1}=300\right)<\bar{Y}_{S}\left(R d_{0}=30\right) .
$$

The differences between the $\bar{Y}_{s}$ values provided by different radiation models are greater than those observed in figure 4 for $\bar{Z}_{s}$. For example, the maximum value of the relative difference between $\bar{Y}_{S}(R d=0)$ and $\bar{Y}_{S}\left(R d_{0}=30\right) / \bar{Y}_{S}\left(R d_{1}=300\right)$ is $32 \% / 26 \%$ and occurs at small $R e$ 
number values. The relative differences between the results provided by different $S P_{\mathrm{N}}$ approximations are smaller. The maximum relative difference between $\bar{Y}_{S}\left(R d_{0}=30\right)$ and $\bar{Y}_{S}\left(R d_{1}=300\right)$ is around $12 \%$.

Figure 6 shows that thermal radiation influences significantly the average $N u$ values. The increase in $N u$ for $P_{0}$ and $P_{1}$ approximations is mainly given by the decrease in the driving force. Thermal radiation is responsible for the decrease in the driven force. The driven force for $P_{1}$ approximation is three to four times smaller than the driven force of the no-radiation model. For $P_{0}$ approximation, the driving force is ten times smaller than the driven force for $P_{1}$ approximation. Figure 6 also shows that the contribution of the surface radiation heat flux to $\mathrm{Nu}$ number decreases with the increase in $R e$.

Figure 7 shows that thermal radiation has no influence on the average $S h$ number values. The increase in $R e$ increases the $S h$ number values. Also, the present $N u(R d=0)$ and $S h$ values agree very well to the $N u$ and $S h$ values computed with relations 3-49 $(R e=0.1)$ and 5-25 $(R e>$ 0.1) from [24]. Figure 8 shows that the external effectiveness factor increases with the increase in $R e$ and, for constant $R e$, thermal radiation decreases the external effectiveness factor. For a given $R e$ number, the numerical values of the external effectiveness factor verify the relation,

$$
\eta_{e}(R d=0)>\eta_{e}\left(R d_{1}=300\right)>\eta_{e}\left(R d_{0}=30\right) .
$$

The third set of dimensionless reaction parameters was computed considering $T_{\infty}=500 \mathrm{~K}$. The values of $B, \gamma$ and $\varphi$ are, $B=0.45, \gamma=14.0, \varphi=3.0$. Considering $\lambda=0.071 \mathrm{~W} \mathrm{~m}^{-1} \mathrm{~K}^{-1}$, the value obtained for $R d_{1}$ is $R d_{1}=400$. The value assumed to $R d_{0}$ is $R d_{0}=40$.

Figure 9 shows that the differences between the values of $\bar{Z}_{s}$ computed with different radiation models are higher than those observed in figure 4. For all models, the increase in $R e$ increases the sphere surface temperature. The largest values of the surface temperature are provided by the no-radiation model while the smallest values of the surface temperature are given by $P_{0}$ approximation. The $\bar{Z}_{s}$ values computed with $P_{1}$ approximation are greater than the values of the $P_{0}$ model but little than the values of the no-radiation model. The increase in $R e$ increases the differences between the results provided by $P_{0}$ and $P_{1}$ approximations.

Figure 10 shows the values of sphere surface average dimensionless conversion, $\bar{X}_{s}$. As expected, the differences between the values of the sphere surface temperature generate larger differences between the values of the sphere surface conversion. For the no-radiation model the transformation is almost complete. The surface conversion values computed with $P_{1}$ 
approximation are relatively high and do not change significantly with the increase in $R e$. For the $P_{0}$ approximation, the increase in $R e$ decreases strongly the values of the surface conversion. In this case, the increase in the mass transfer rate with the increase in $R e$ is higher than the increase in the surface reaction rate.

The influence of the $R e$ number and thermal radiation model on the average $\mathrm{Nu}$ and $\mathrm{Sh}$ numbers is similar to that presented in figure 6 and 7. Thermal radiation has no influence on the average $S h$ number values and the present $N u(R d=0)$ and $S h$ values agree very well to the $N u$ and $S h$ values computed with relations 3-49 $(R e=0.1)$ and 5-25 $(R e>0.1)$ from [24]. Figure 11 shows that the external effectiveness factor increases with the increase in $R e$. Significant differences occur only between $P_{0}$ and $P_{1}$ approximations for $R e$ values greater than 10 .

The results presented in this section were obtained for the same values of the radiation parameters $k_{a}, \beta$ and $\varepsilon$. The effect of these parameters on the surface chemical reaction was analysed and discussed in [22]. The results obtained in [22] were certified by the present numerical simulations., i.e., the increase in the absorption coefficient $k_{a}$ and the decrease in the total attenuation factor $\beta$ and the emissivity coefficient $\varepsilon$ increase the effect of thermal radiation.

\section{Conclusions}

The effect of thermal radiation on the steady-state, heat and mass transfer from a fluid flow to a sphere when an exothermic chemical reaction takes place on the surface of the sphere is analysed in the present work. The radiative transfer is modeled by the diffusion approximations $P_{0}$ and $P_{1}$.

The results presented in the previous section show that Prater number is the key parameter for the process investigated in this work. The effect of the thermal radiation depends on the temperature variation. The temperature variation depends on the values of the Prater number. For very small values of the Prater number the system is quasi-isotherm and the effect of thermal radiation is not significant. For moderate and large values of the Prater number, the effect of thermal radiation on the surface concentration and temperature cannot be neglected. The neglection of the thermal radiation can induce significant errors in the computation of the kinetic parameters. 


\section{References}

[1] P. M. Chung, Chemically reacting nonequilibrium boundary layers, Advances in Heat Transfer 2 (1965) 109 - 270.

[2] N.C. Freeman, P.G. Simpkins, On the diffusion of species in similar boundary layers with finite recombination rate at the wall, Quart. J. Mech. Appl. Math. 18 (1965) 213 - 229.

[3] H. Fox, P.A. Libby, Dissociated laminar boundary layers with heterogeneous recombination, Phys. Fluids 9 (1966) 33.

[4] R. Mihail, A superposition integral equation for catalytic external surfaces, Chem. Eng. Sci. 25 (1970) $463-474$.

[5] R. Mihail, External catalytic reactions in non-Newtonian fluids, Chem. Eng. Sci. 26 (1971) 2120 - 2121.

[6] R. Mihail, Shape effects in external surface catalysis, Chem. Eng. Sci. 27 (1972) 845 - 854.

[7] Y.P. Gupalo, Y.S. Ryazantsev, Mass and heat transfer from a sphere in laminar flow, Chem. Eng. Sci. 27 (1972) 61 - 68.

[8] Y.P. Gupalo, A.D. Polyanin, Y.S. Ryazantsev, Y.A. Sergeev, Convective diffusion to a solid particle in gas flow at non-linear kinetics of the heterogeneous chemical reaction, Dokl. Akad. Nauk. SSSR. 237 (1977) 86 - 89.

[9] A.D. Polyanin, Y.A. Sergeev, Convective diffusion to a reacting in a fluid. Nonlinear surface reaction kinetics, Int. J. Heat Mass Transf. 23 (1980) 1171 - 1182.

[10] R.W. Schefer, Catalyzed combustion of $\mathrm{H}_{2} /$ Air mixtures in a flat plate boundary layer: II. Numerical model, Combustion Flame 45 (1982) 171 - 190.

[11] P. Markatou, L.D. Pfefferle, M.T. Smooke, A computational study of methane-air combustion over heated catalytic and non-catalytic surfaces, Combustion Flame 93 (1993) 185 - 201.

[12] T. Elperin, A. Fominykh, Exact analytical solution of a convective diffusion from a wedge to a flow with a first order chemical reaction at the surface, Int. Comm. Heat Mass Transf. 21 (1994) $227-235$.

[13] T. Elperin, A. Fominykh, Mass transfer between a two-dimensional wall jet with a heterogeneous chemical reaction of the first order and a wall: analytical solution, Int. J. Heat Mass Transf. 39 (1996) $3287-3291$. 
[14] T. Elperin, A. Fominykh, Z. Orenbakh, Mass transfer with heterogeneous chemical reaction of the first-order in Falkner-Skan flow of a power-law fluid, Chem. Eng. Comm. 192 (2005) 1505 $-1514$.

[15] J.H. Merkin, A model for isothermal homogeneous-heterogeneous reactions in boundarylayer flow, Math. Comput. Model. 24 (1996) 125 - 136.

[16] Gh. Juncu, R. Mihail, Multigrid solution of the diffusion - convection - reaction equations which describe the mass and/or heat transfer from or to a spherical particle, Comput. Chem. Eng. 13 (1989) $259-270$.

[17] W.R. Paterson, Mass transfer to, and reaction on, a sphere immersed in a stationary or flowing gas, Chem. Eng. Sci. 55 (2000) 3567 - 3570.

[18] R.C. Lindberg, R.A. Schmitz, On the multiplicity of steady states in boundary layer problems with surface reaction, Chem. Eng. Sci. 24 (1969) 1113 - 1129.

[19] R.C. Lindberg, R.A. Schmitz, Multiplicity of steady states with surface reaction on a blunt object in a convective system, Chem. Eng. Sci. 25 (1970) $901-904$.

[20] R. Mihail, C. Teodorescu, Laminar boundary layer with nonisothermal surface reaction, Chem. Eng. Sci. 30 (1975) 993 - 996.

[21] A. Linan, C. Trevino, Ignition and extinction of catalytic reactions on a flat plate, Combustion Sci. Tech. 38 (1984) $113-128$.

[22] Gh. Juncu, The effect of thermal radiation on the surface catalytic chemical reaction, Rev. Chimie 71 (2020) 1 - 10.

[23] E.W. Larsen, G. Thömmes, A. Klar, M. Seaïd, T. Götz, Simplified $P_{N}$ approximations to the equations of radiative heat transfer and applications, J. Comput. Phys. 183 (2002) 652 - 675.

[24] M.F. Modest, Radiative Heat Transfer, $3^{\text {rd }}$ ed., Academic Press, Oxford, 2013.

[25] R.G. McClarren, Theoretical aspects of the simplified $P_{\mathrm{n}}$ equations, Transp. Theory Statis. Phys. 39 (2011) 73 - 109.

[26] Gh. Juncu, R. Mihail, Numerical solution of the steady incompressible Navier-Stokes equations for the flow past a sphere by a multigrid defect correction technique, Int. J. Num. Meth. Fluids 11 (1990) $379-395$.

[27] P. Wesseling, Principles of Computational Fluid Dynamics, Springer, Berlin, 2001.

[28] R. Clift, J.R. Grace, M.E. Weber, Bubbles, Drops and Particles, New York: Academic Press, 1978. 
[29] T.A. Johnson, V.C. Patel, Flow past a sphere up to a Reynolds number of 300, J. Fluid Mech., 378 (1999) $19-70$.

[30] L. Wang, T.P. Tran, D.V. Vo, M. Sakurai, H. Kameyama, Design of novel Pt-structured catalyst on anodic aluminum support for VOC's catalytic combustion, Applied Catalysis A: General 350 (2008) $150-156$.

[31] C. He, J. Cheng, X. Zhang, M. Douthwaite, S. Pattisson, Z. Hao, Recent advances in the catalytic oxidation of volatile organic compounds: a review based on pollutant sorts and sources, Chemical Reviews 119 (2019) 4471 - 4568.

[32] C. Yang, G. Miao, Y. Pi, Q. Xia, J. Wu, Z. Li, J. Xiao, Abatement of various types of VOCs by adsorption/catalytic oxidation: A review, Chem. Eng. J. 370 (2019) 1128 - 1153.

[33] Gh. Juncu, J.P. Brouwer, Alette Mulder, Sensitivity analysis of the methane catalytic combustion in a sinter metal reactor with integrated heat exchanger, Chem. Eng. Sci. 51 (1996) 3101-3106.

[34] Gh. Juncu, J.P. Brouwer, Alette Mulder, Numerical modeling of the methane catalytic combustion in a sinter metal reactor with integrated heat exchanger, Int. J. Comp. Fluid Dynamics 8 (1997) 221-234.

[35] L. He, Y. Fan, J. Bellettre, J. Yue, L. Luo, A review on catalytic methane combustion al low temperatures: Catalysts, mechanisms, reaction conditions and reactor designs, Renewable and Sustainable Energy Reviews 119 (2020) 109589. 


\section{Figures Caption}

Figure 1. Schematic of the problem.

Figure 2. Variation of the surface average dimensionless concentration with $\operatorname{Re}$ for $B=0.85, \gamma$ $=18.0$ and $\varphi=0.06\left(a=0.01 \mathrm{~m}, k_{a}=1 \mathrm{~m}^{-1}, \beta=3 \mathrm{~m}^{-1}\right.$ and $\left.\varepsilon=0.7\right)$.

Figure 3. Values of the surface average dimensionless temperature function of $\operatorname{Re}$ for $B=0.85, \gamma$ $=18.0$ and $\varphi=0.16\left(a=0.01 \mathrm{~m}, k_{a}=1 \mathrm{~m}^{-1}, \beta=3 \mathrm{~m}^{-1}\right.$ and $\left.\varepsilon=0.7\right)$.

Figure 4. Values of the surface average dimensionless temperature function of $\operatorname{Re}$ for $B=0.05, \gamma$ $=10.0$ and $\varphi=6.4\left(a=0.01 \mathrm{~m}, k_{a}=1 \mathrm{~m}^{-1}, \beta=3 \mathrm{~m}^{-1}\right.$ and $\left.\varepsilon=0.7\right)$.

Figure 5. Variation of the surface average dimensionless concentration with $\operatorname{Re}$ for $B=0.05, \gamma$ $=10.0$ and $\varphi=6.4\left(a=0.01 \mathrm{~m}, k_{a}=1 \mathrm{~m}^{-1}, \beta=3 \mathrm{~m}^{-1}\right.$ and $\left.\varepsilon=0.7\right)$.

Figure 6. The influence of the $R e$ number on the average $N u$ number for $B=0.05, \gamma=10.0, \varphi=6.4$, $\left(a=0.01 \mathrm{~m}, k_{a}=1 \mathrm{~m}^{-1}, \beta=3 \mathrm{~m}^{-1}\right.$ and $\left.\varepsilon=0.7\right)$.

Figure 7. The influence of the Re number on the average $S h$ number for $B=0.05, \gamma=10.0, \varphi=6.4$, ( $a=0.01 \mathrm{~m}, k_{a}=1 \mathrm{~m}^{-1}, \beta=3 \mathrm{~m}^{-1}$ and $\varepsilon=0.7$ ).

Figure 8. The influence of the $R e$ number on the external effectiveness factor for $B=0.05, \gamma=10.0$, $\varphi=6.4,\left(a=0.01 \mathrm{~m}, k_{a}=1 \mathrm{~m}^{-1}, \beta=3 \mathrm{~m}^{-1}\right.$ and $\left.\varepsilon=0.7\right)$.

Figure 9. Values of the surface average dimensionless temperature function of $\operatorname{Re}$ for $B=0.45, \gamma$ $=14.0$ and $\varphi=3.0\left(a=0.01 \mathrm{~m}, k_{a}=1 \mathrm{~m}^{-1}, \beta=3 \mathrm{~m}^{-1}\right.$ and $\left.\varepsilon=0.7\right)$.

Figure 10. Variation of the surface average dimensionless conversion with $\operatorname{Re}$ for $B=0.45, \gamma=14.0$ and $\varphi=3.0\left(a=0.01 \mathrm{~m}, k_{a}=1 \mathrm{~m}^{-1}, \beta=3 \mathrm{~m}^{-1}\right.$ and $\left.\varepsilon=0.7\right)$.

Figure 11. Variation of the external effectiveness factor with $\operatorname{Re}$ for $B=0.45, \gamma=14.0, \varphi=3.0,(a$ $=0.01 \mathrm{~m}, k_{a}=1 \mathrm{~m}^{-1}, \beta=3 \mathrm{~m}^{-1}$ and $\left.\varepsilon=0.7\right)$. 


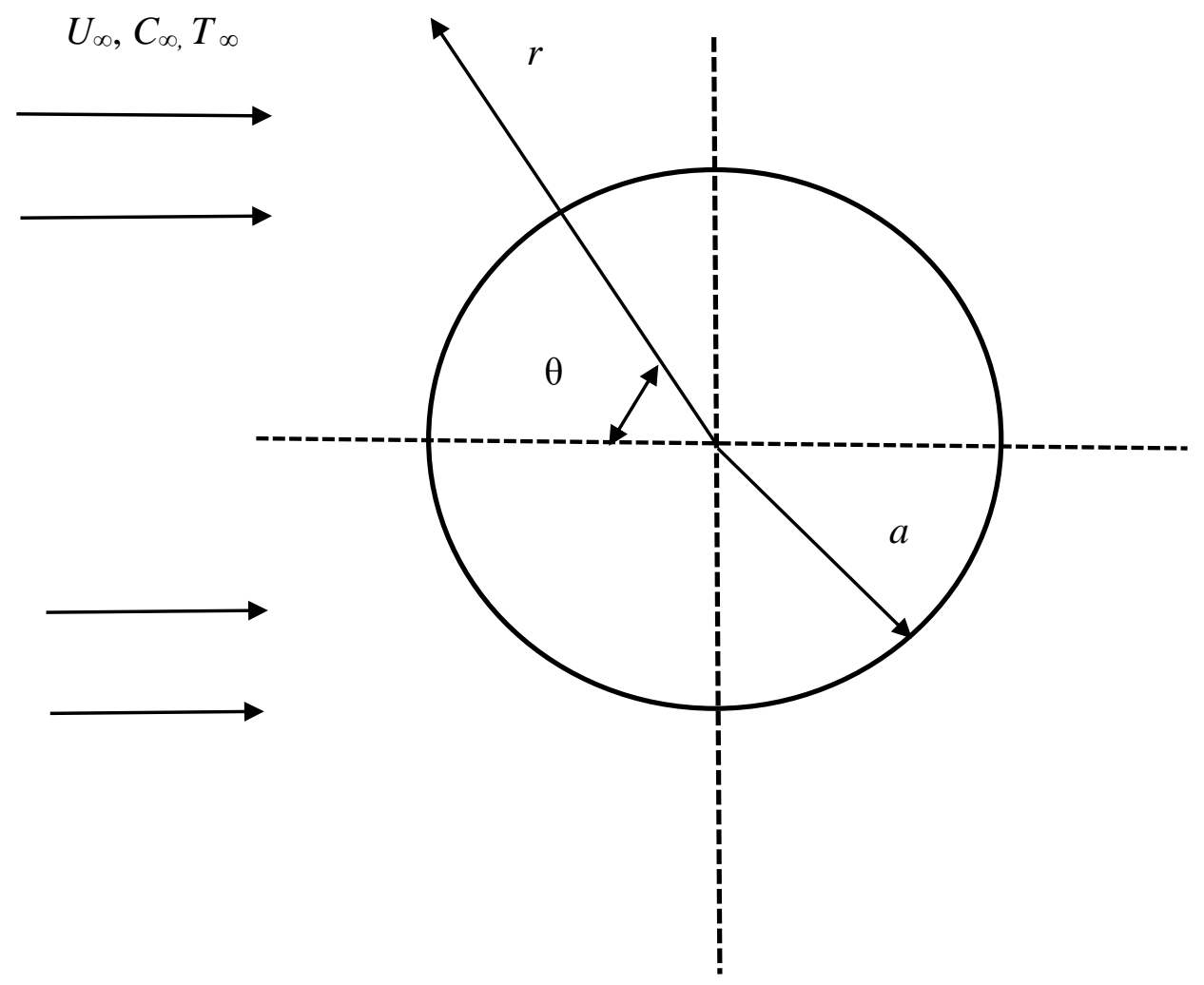

Figure 1. 


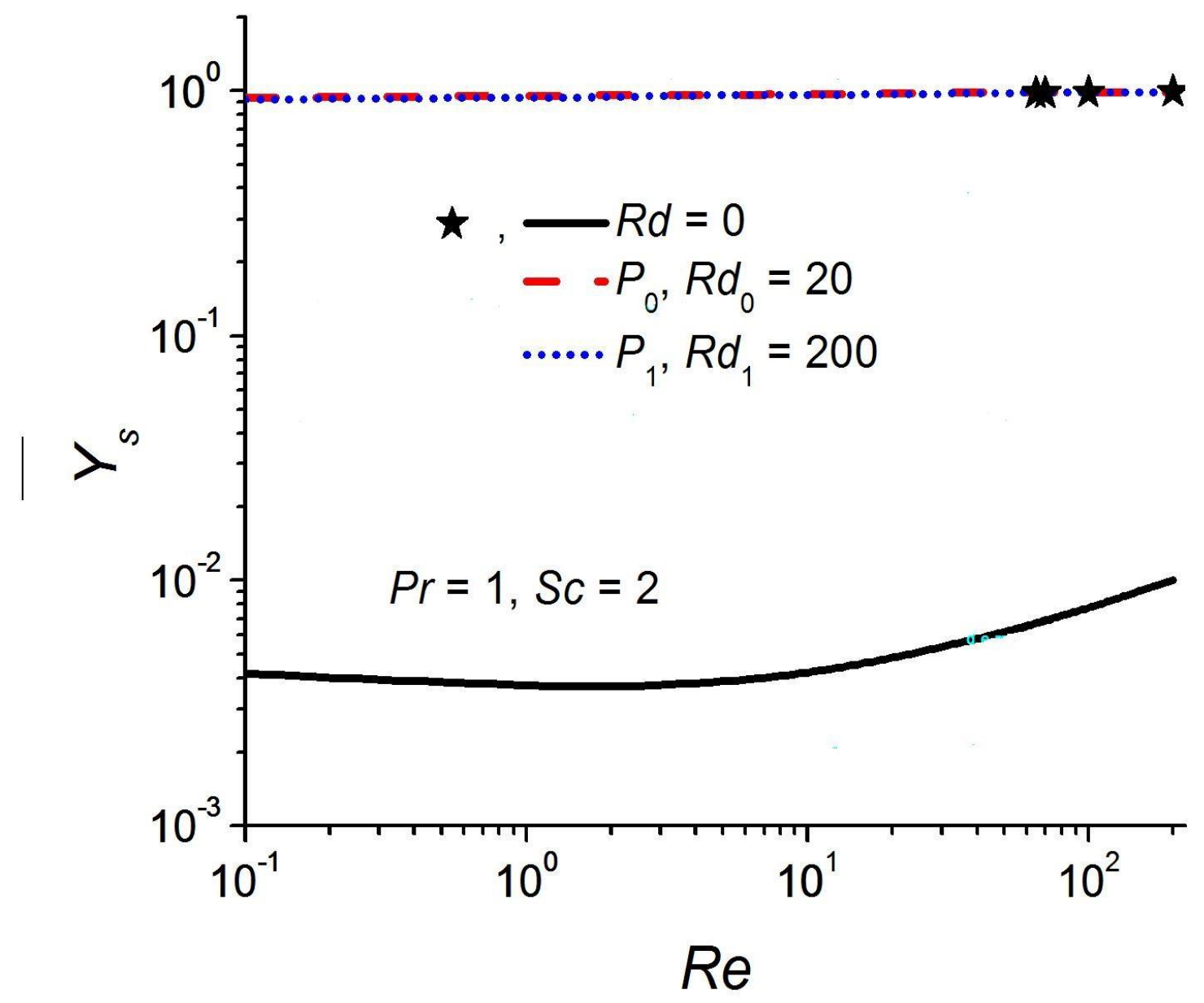

Figure 2. 


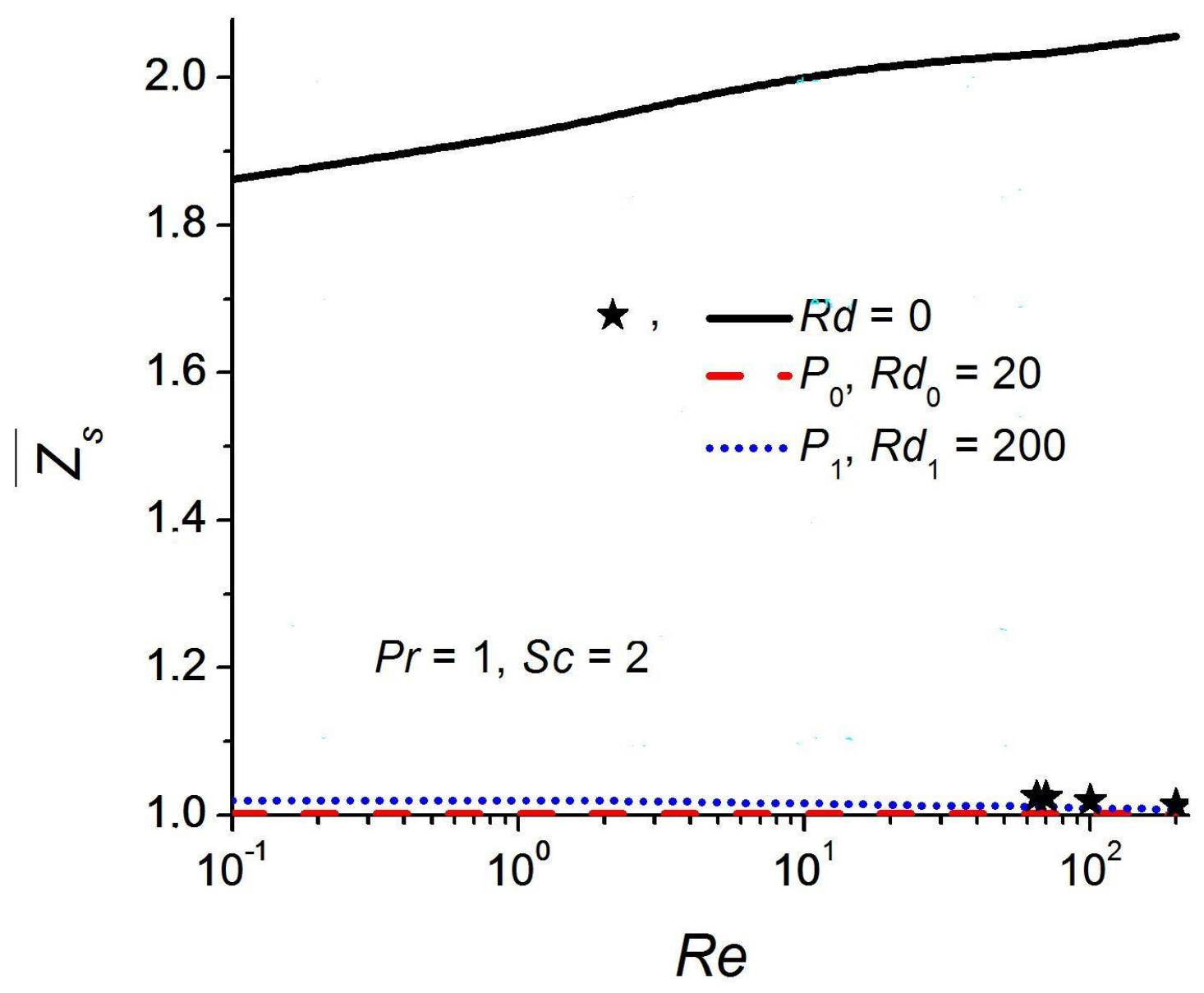

Figure 3. 


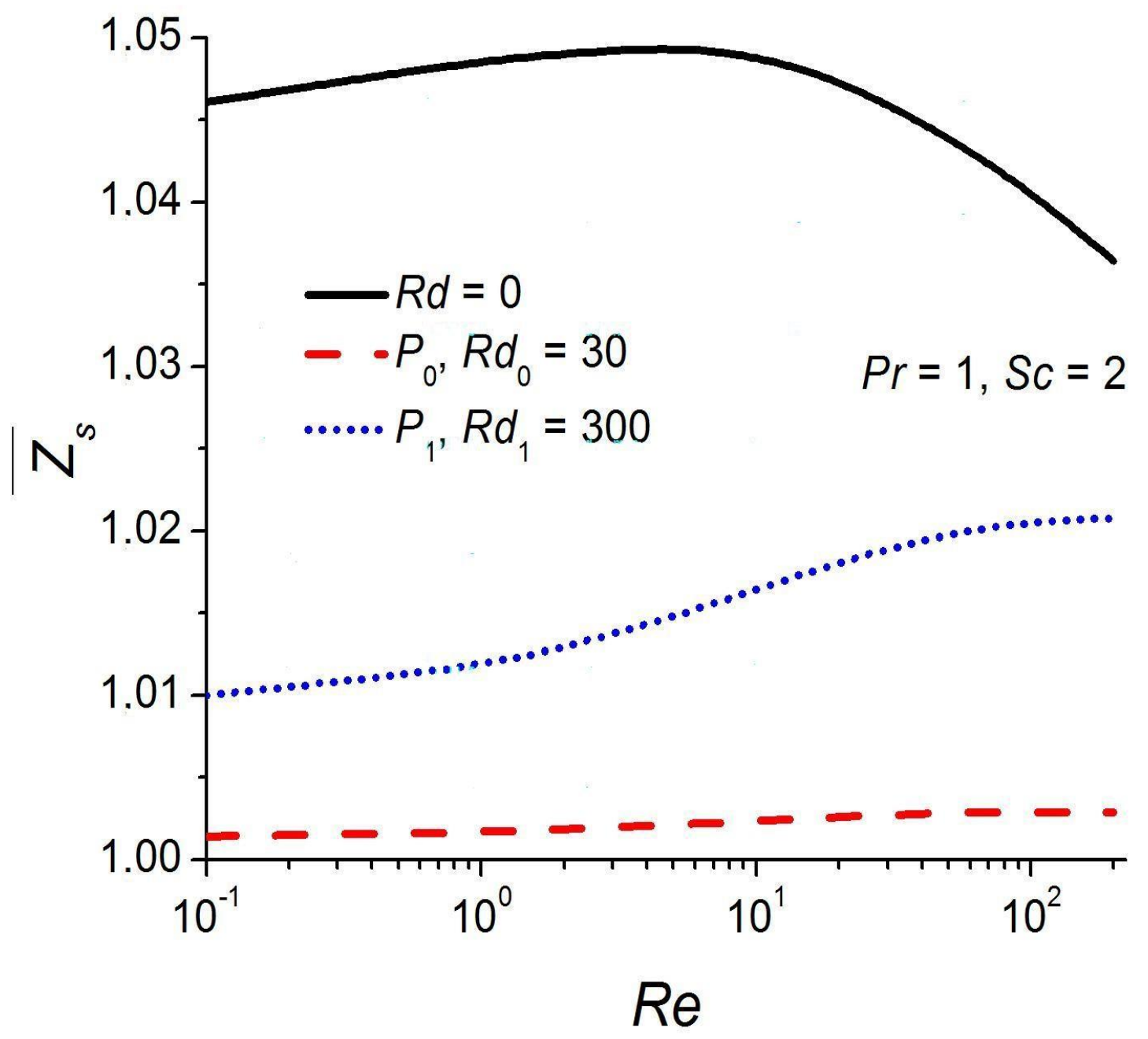

Figure 4. 


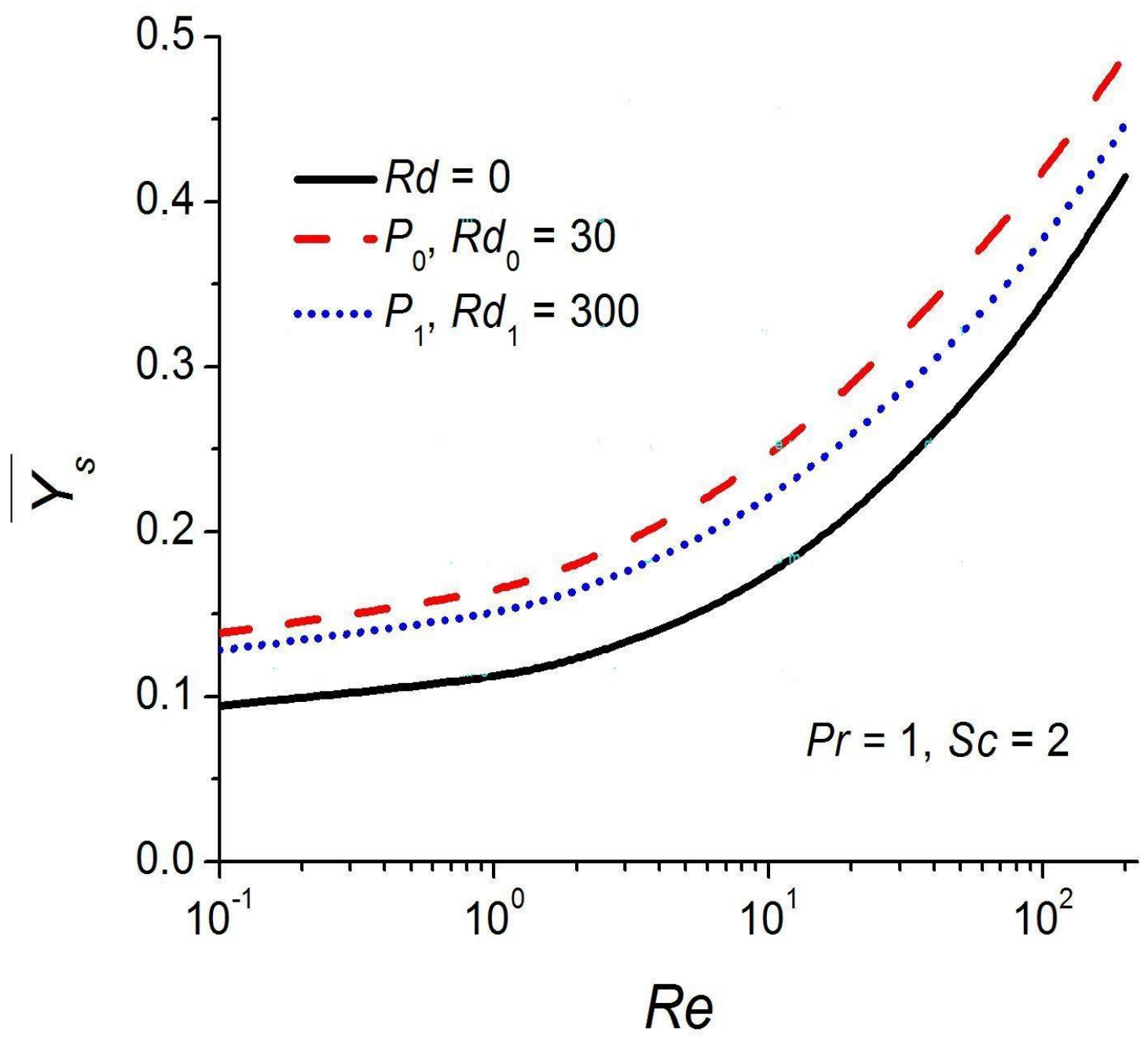

Figure 5. 


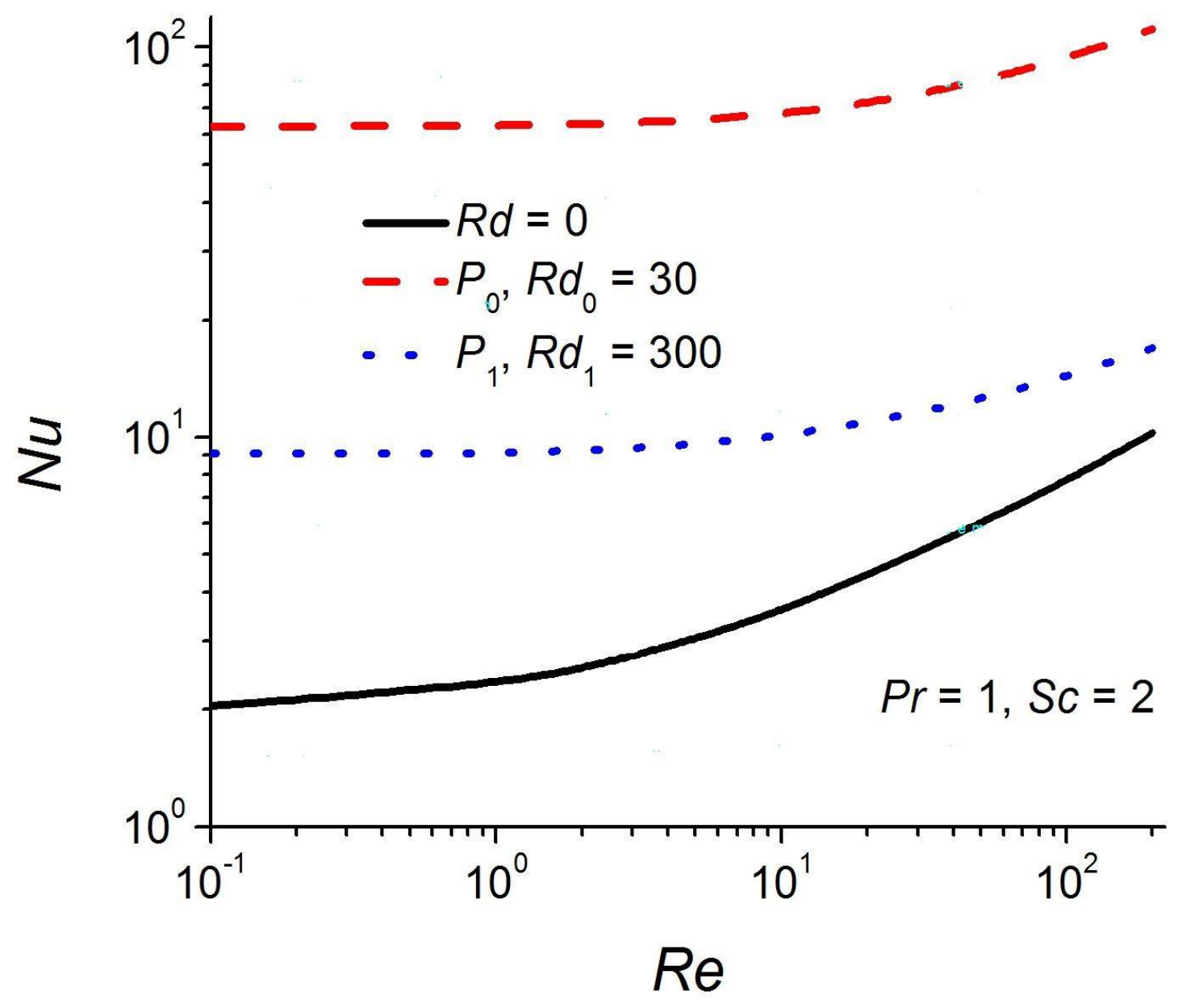

Figure 6. 


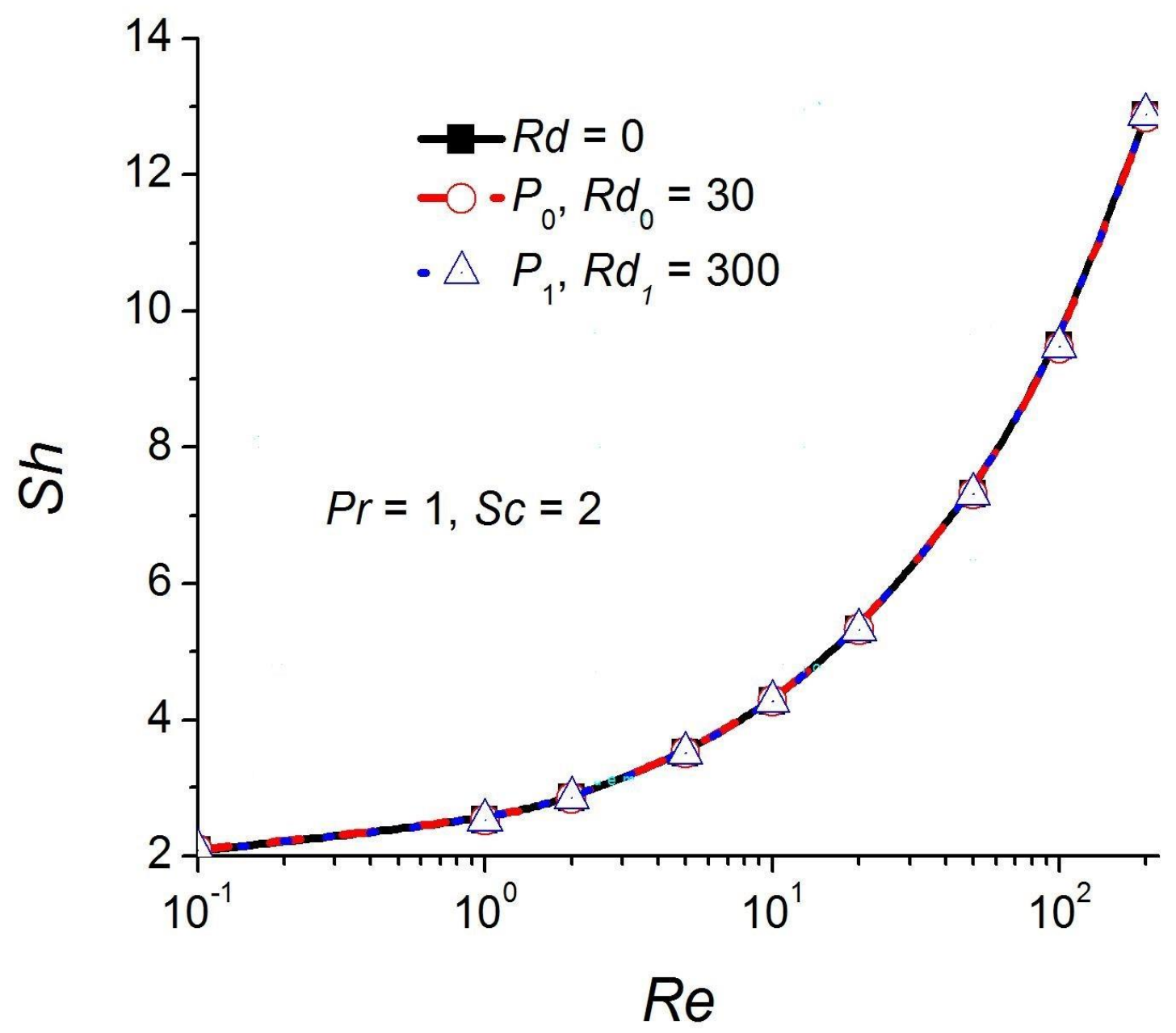

Figure 7. 


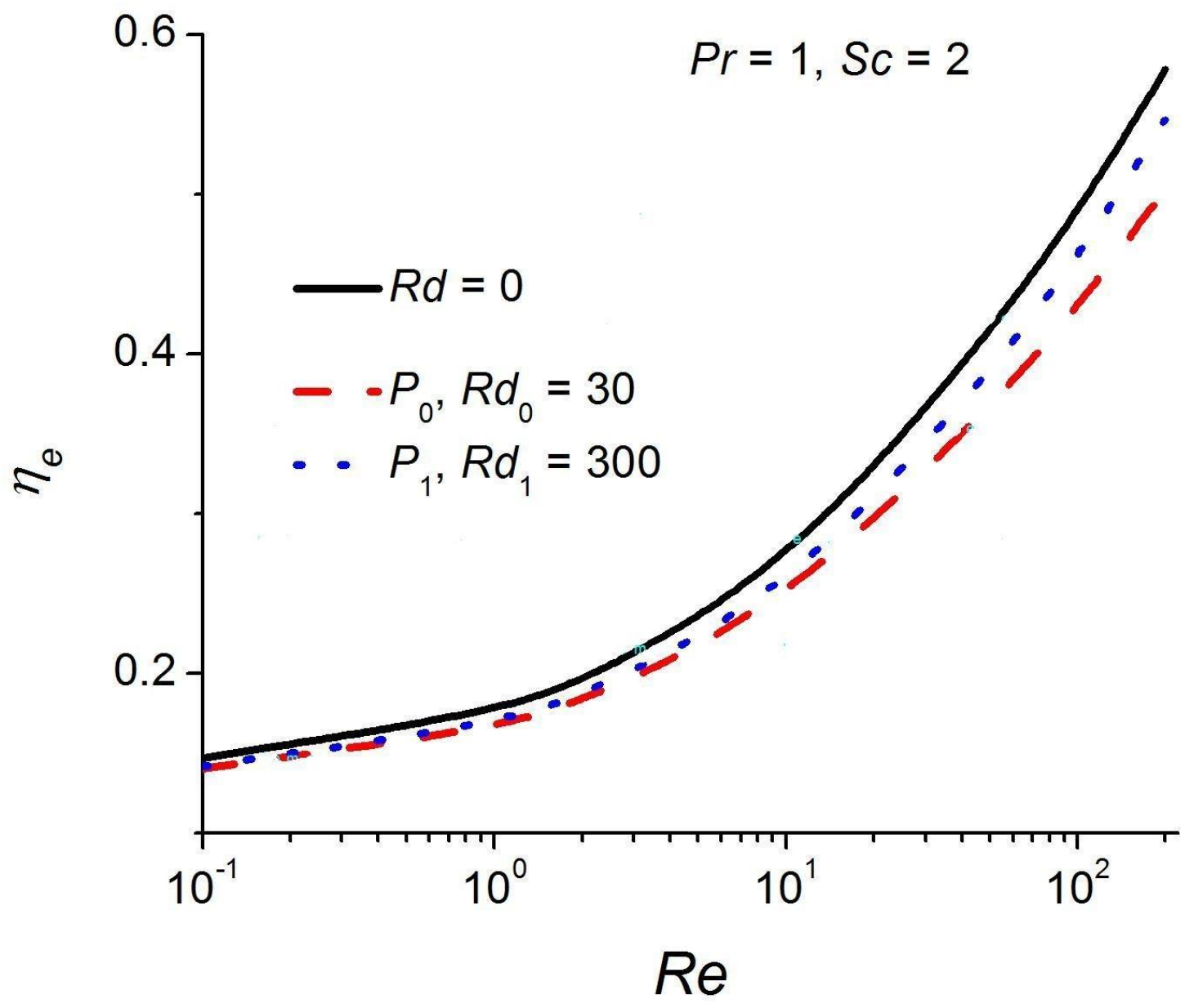

Figure 8. 


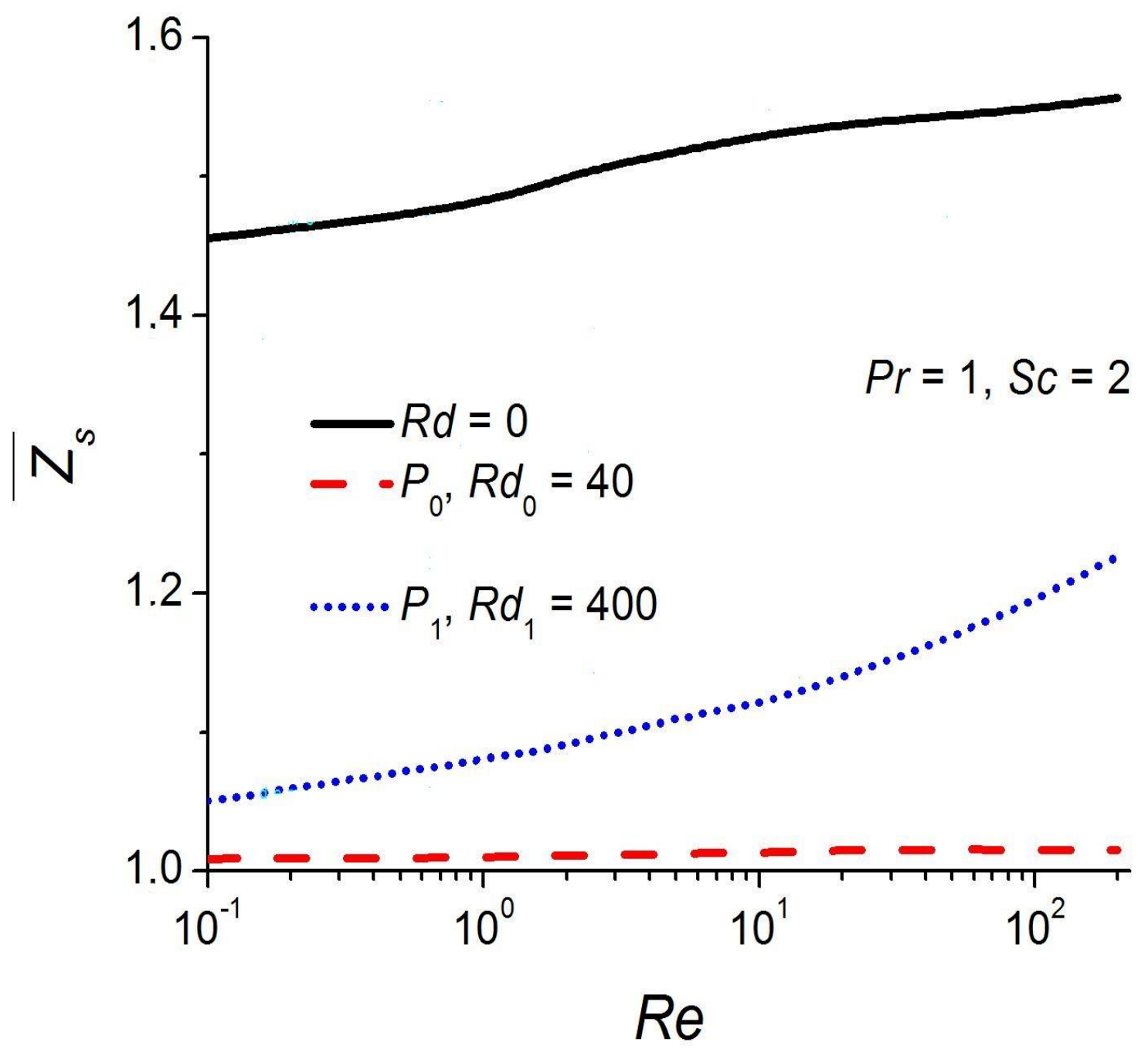

Figure 9. 


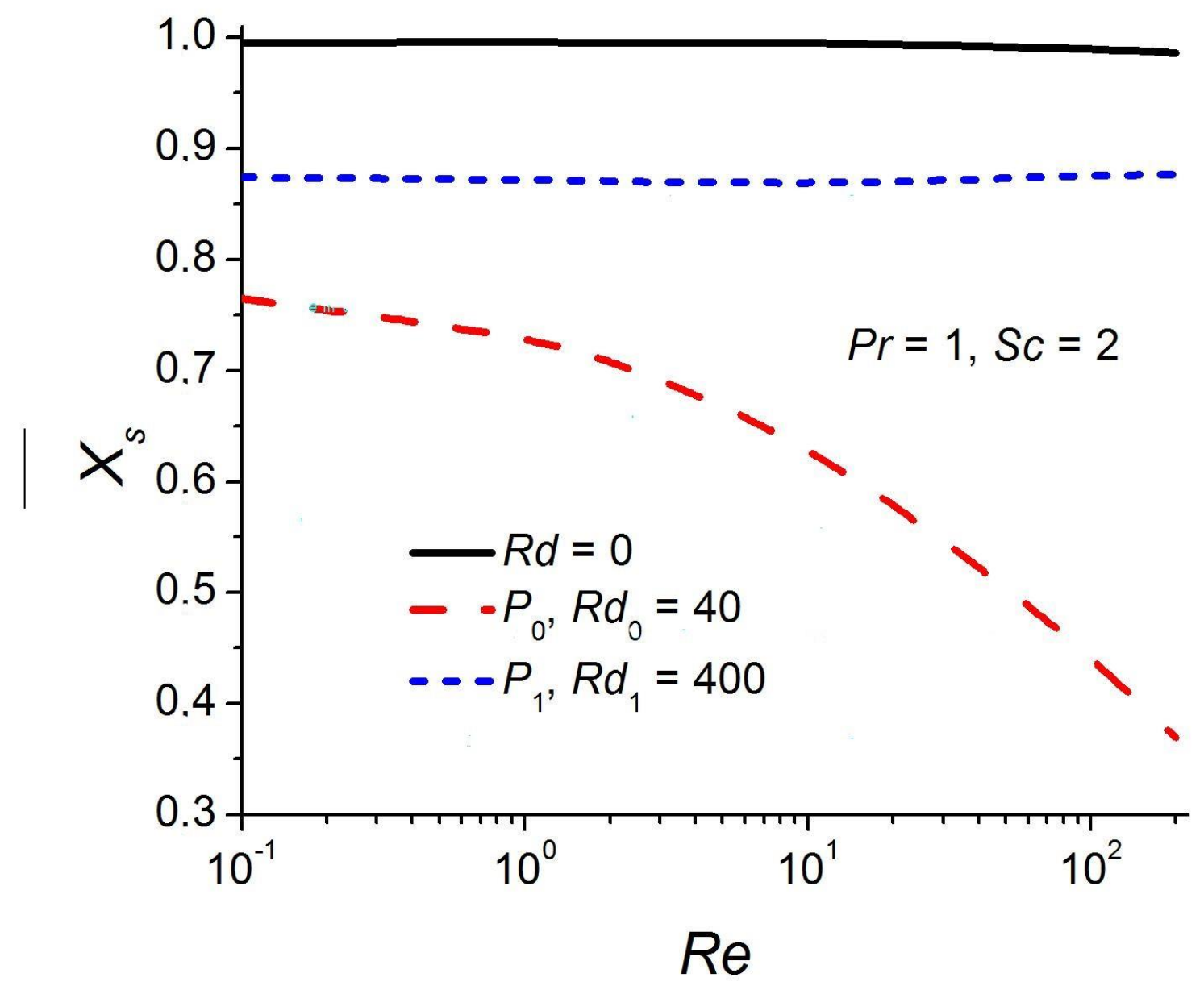

Figure 10. 


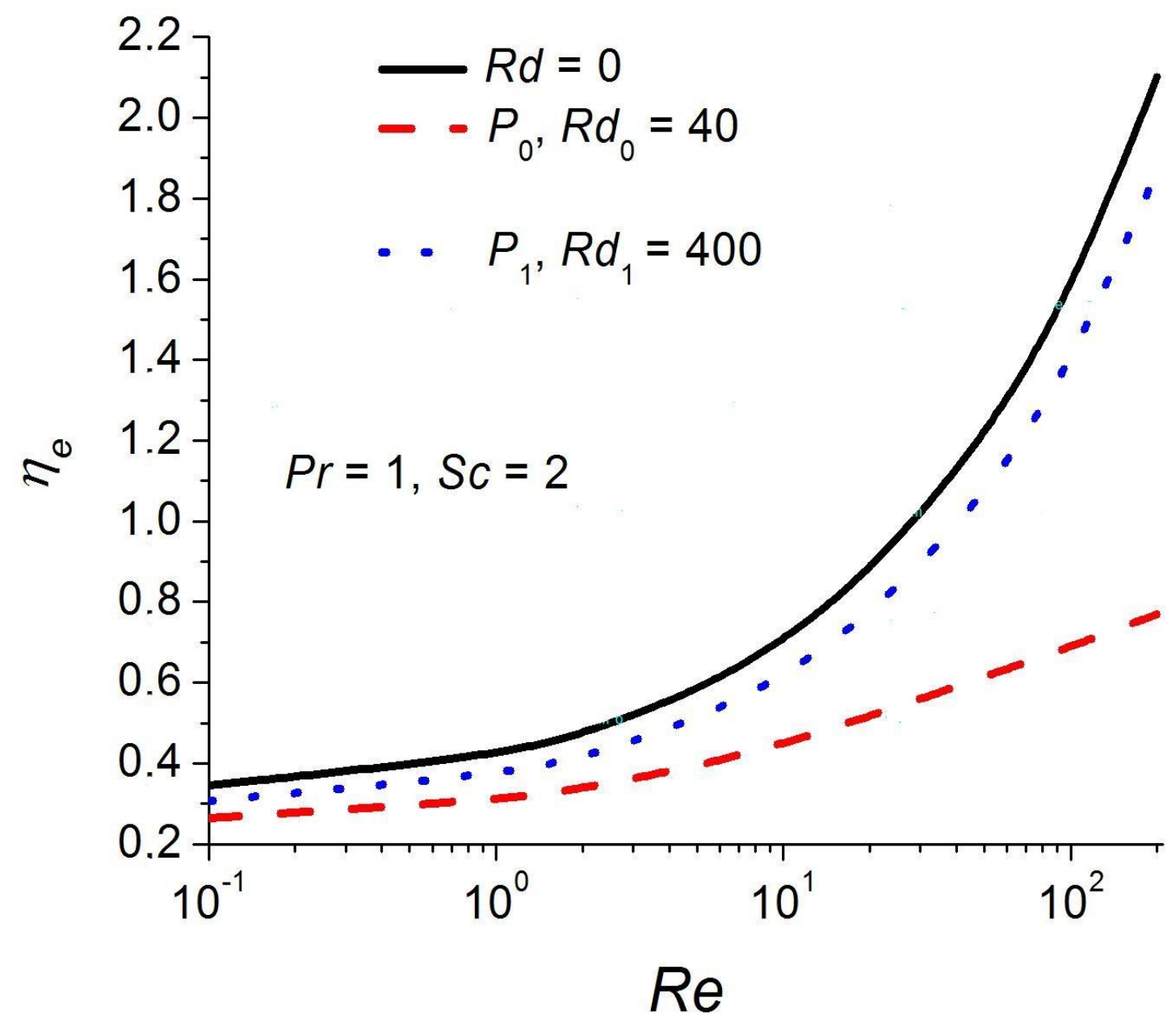

Figure 11. 